\title{
基于几何误差不确定性的滚动导轨运动误差研究 ${ }^{*}$
}

\author{
马雅丽 李阳阳 \\ (大连理工大学机械工程学院 大连 116024)
}

\begin{abstract}
摘要: 产品设计阶段, 滚动导轨几何误差是未知的, 具有不确定性, 其运动精度亦是不确定的。为了研究设计阶段滚动导 轨几何误差不确定对其运动误差的影响规律, 提出将不确定的几何误差等效为滚动体弹性变形的运动误差分析方法。采用 区间参数和小位移旋量描述滚动导轨的几何误差, 并采用蒙特卡罗模拟(Monte Carlo simulation, MCS)方法进行不确定模拟 仿真, 获得满足约束的几何误差分布范围。基于此, 将滚动导轨几何误差等效为滚动体的弹性变形并根据赫兹接触理论建 立滚动导轨的静力平衡方程, 从而实现了滚动导轨运动误差的不确定性分析。以某一滚动导轨为例, 基于提出的方法分析 了滚动导轨几何误差不确定性下其运动误差的分布规律, 并分析了不同直线度公差、预紧力以及外载荷对导轨运动误差的 影响规律。
\end{abstract}

关键词: 几何误差；不确定性；滚动导轨；运动误差；蒙特卡洛模拟方法

中图分类号: TH161

\section{Motion Error of Rolling Guide Based on Uncertainty of Geometric Error}

\author{
MA Yali LI Yangyang \\ (College of Mechanical Engineering, Dalian University of Science and Technology, Dalian 116024)
}

\begin{abstract}
In product design stage, the geometric error of rolling guide is unknown and uncertain, and its motion precision is also uncertain. To research the influence of uncertain geometric error on the motion error of rolling guide, an analysis method of motion error with the uncertain geometric error being equivalent to the elastic deformation of rolling balls is proposed. Interval parameters and small displacement torsor are used to describe the uncertainties of geometric error, and Monte Carlo simulation (MCS) method is used to obtain distribution range of geometric error restricted by constraint inequalities. Taking the distribution range of geometric error torsor parameters as modelling samples, static force equilibrium equations of rolling guide system are established with geometrical error and motion error of rolling guide being equivalently mapped into the elastic deformation of the rolling elements via the Hertz contact theory. With a case of certain rolling guide, the changing rules of rolling guide motion error in the conditions of uncertainty of straightness error of rolling guide are obtained, and the effects of straightness tolerance, preloads and external loads under different design requirements on motion error are analysed.
\end{abstract}

Key words: geometric error; uncertainty; rolling guide; motion error; Monte Carlo simulation method

\section{0 前言}

中国制造 2025 将高档数控机床列为十大重点 领域, 对其提出了高精密、高效的发展要求。因此, 数控机床精度设计与提高变得日益重要。数控机床 组成零部件的各类误差对机床整体精度影响程度不 同, 且各误差随多种影响因素变化。因此, 在机床 设计阶段分析零部件各类误差的影响因素及其影响 情况可有效指导机床的精度设计。

* 国家科技重大专项资助项目(2015ZX04014-021)。20180405 收到初稿, 20181116 收到修改稿
滚动导轨是机床重要的部件, 其运动误差, 即 运动部件运动时, 功能点的实际位置、轨迹、速度 等与理想状态的偏差 ${ }^{[1]}$, 直接影响着机床的加工精 度。基于多体系统理论，程强等 ${ }^{[2]}$ 在敏感度分析基 础上提出了关键性几何误差源识别的方法，该方法 可以有效地识别对机床空间误差影响较大的零部件 几何误差因素。胡鹏浩等 ${ }^{[3]}$ 采用双频激光干涉仪获 取滑块直线度误差，建立了滑块运动直线度对 3-PUU 并联坐标测量机的运动误差模型, 并分析了 直线度误差对运动误差的贡献率。要小鹏等 ${ }^{[4]}$ 研究 不同工况下温度场与定位误差的变化规律，采用灰 色关联度算法建立定位误差敏感矩阵对机床几何基 
准误差和热误差进行解耦分离建模。国外方面, 结 合赫兹接触理论和有限元方法, OHTA 等 ${ }^{[5]}$ 考虑导 轨和滚动体的弹性变形建立了滚动导轨弹性变形模 型。JEONG 等 ${ }^{[6]}$ 建立了 8 个弹簧等效模型联接运动 部件和导轨, 并采用有限元法分析了导轨系统的动 静态特性。KHIM 等 ${ }^{[7]}$ 建立导轨的几何误差形式和 回复力之间的传递函数并建立运动过程中的平衡方 程用于分析导轨五自由度的运动误差。鉴于导轨的 几何误差形式不易获取, KHIM 等 $^{[8]}$ 又进一步采用 工作台的直线度误差替代导轨的几何误差, 并分析 导轨的运动误差。

然而, 滚动导轨的几何误差往往是不确定的, 造成滚动导轨运动误差的不确定, 因此考虑几何误 差的不确定性进行滚动导轨运动误差分析更符合工 程实际。不确定分析是研究各种不确定的系统参数 影响系统性能的规律。RACKWITZ ${ }^{[9]}$ 最早提出了一 种不确定分析方法应用于当系统性能函数连续可 导, 参数分布函数已知时的情况, 分析了随机载荷 作用下的结构可靠性。ZHOU 等 ${ }^{[10]}$ 在汽车零部件稳 健性优化设计中提出了四阶矩分析方法解决了零件 参数分布函数不规则或者不确定的问题。针对复杂 系统中存在的多元不确定性和多层嵌套问题, 刘成 武等 ${ }^{[11]}$ 提出了随机和区间不确定性的序列化多学 科可靠性设计优化方法和混合可靠性评价指标。吕 辉等 ${ }^{[12]}$ 将参数不确定性引入到汽车盘式制动器的 振动稳定性分析中, 采用响应面法和有限元复特征 值技术建立包含随机参数和区间参数的制动器系 统不稳定特征值的响应面近似模型, 进而对汽车盘 式制动器系统进行参数灵敏度和可靠性分析。黎凯 等 ${ }^{[13]}$ 综合考虑参数和代理模型的不确定性, 构建了 新的冲压稳健性设计方法。当系统参数分布函数未 知但系统的标准差、均值或者变化区间已知, 或者 系统性能函数不可导时, 常用的方法是采样统计 法, 如蒙特卡罗模拟、超立方采样法和正交表法等。 针对稳健性设计中代理模型不确定性的问题, 熊芬 芬 ${ }^{[14]}$ 提出了一种基于蒙特卡罗抽样的量化方法, 在 传统参数不确定性的基础上又考虑了代理模型的 不确定性。余治民等 ${ }^{[15]}$ 提出了蒙特卡罗模拟与响应 面法相结合的公差建模方法模拟实际公差表面的 变动, 以平面尺寸和平面度公差为对象获取公差变 动要素时间变动区间。崔鍂否等 ${ }^{[16]}$ 采用蒙特卡罗模 拟方法研究 6-UPS 并联机构, 在 42 项误差源中选 取支杆长度误差和轴承游隙对机构动平台位姿误 差的影响。

上述成果奠定了滚动导轨运动误差分析的算法 依据和理论基础, 但是多基于设计参数确定性分
析, 存在着分析结果适应性差、可靠性低等缺点。 因此采用不确定性分析方法分析导轨运动误差, 以区间参数和小位移旋量描述滚动导轨几何误差 的不确定性, 并基于 MCS 方法模拟滚动导轨几何 误差的实际变动分布范围。基此, 将不确定几何 误差等效为滚动体的弹性变形并根据赫兹接触理 论建立滚动导轨的静力平衡方程, 最终得到运动 误差分析模型。通过某一滚动导轨分析了滚动导 轨几何误差不确定下对运动误差的影响规律, 为 机床精度的设计以及机床组成部件的精度分配提 供理论依据。

\section{1 几何误差不确定性描述}

滚动导轨主要由导轨、滚动体、滑块等零件组 成。滑块沿着导轨方向实现往复直线运动, 其结构 见图 1 。滚动导轨的几何误差是典型几何要素实际 状态与理想状态的偏差, 包括滑块和导轨滚道曲率 半径尺寸, 导轨的直线度误差等, 可以表述为 $X=\left(x_{1}\right.$, $\left.x_{2}, \cdots, x_{n}\right), X$ 为 $n$ 维滚动导轨几何误差变量。滚动 导轨几何误差值在设计阶段是未知的, 具有不确定 性, 而其公差值是约束其变动的区间值。要得到几 何误差的精确分布函数也是很困难的, 但是获得几 何误差不确定量的几何公差则比较容易。采样统计 分析方法往往用来分析分布函数未知时的不确定参 数。因此,采用区间参数和小位移旋量法描述不确定 的几何误差, 并采用 MCS 方法进行模拟仿真, 获 得满足几何公差约束的几何误差分布。
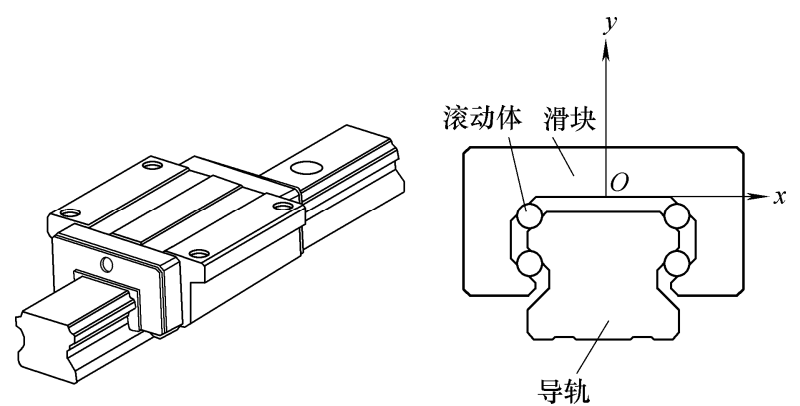

图 1 滚动导轨结构示意图

\section{1 几何误差参数化}

几何误差具有不确定性, 其分布函数也是未知 的, 其参数化的过程就是寻找一些特征参数去表述 几何误差, 进而对不确定几何误差的研究转化为对 特征参数特性的研究。小位移旋量 ${ }^{[17]}$ 是 6 个运动分 量的刚体产生微小位移构成的矢量。三维空间中, 物理量可抽象为点、线、面等基本要素，并有 3 个 沿坐标轴的平动自由度和 3 个绕坐标轴的转动自由 度。分别用 $\boldsymbol{d}=(u, v, w)$ 和 $\boldsymbol{\theta}=(\alpha, \beta, \delta)$ 表示平动和转动 
的微小变动矢量, 则这两组矢量的合成矢量称为小 位移旋量。 $u, v, w, \alpha, \beta, \delta$ 为小位移旋量的旋量 参数。公差域表示公差实际表面脱离名义表面变动 的范围, 而物理表面的基本要素的不确定变动量均 可以通过各自的小位移旋量表示。BOURDET 等 ${ }^{[18]}$ 最早将小位移旋量引入公差领域, 提出基于小位移 旋量的公差数学表示方法, 即用旋量参数的变动来 描述公差实际表面在公差域中的变动。

由于约束的存在, 不同类型公差域的某些旋量 参数应为零。参数设置为零的规则是: 若几何要素 沿某一个自由度方向运动时, 其运动轨迹不导致新 的误差扫掠实体产生。因此, 滚动导轨滑块和导轨 滚道曲率半径几何误差旋量参数可表述为 $(u, v, 0,0$, $0,0)$; 滚动导轨直线度几何误差可以等效为滚动 导轨滚道的曲率中心沿导轨运动方向形成的曲率 轴线的直线度误差, 其旋量参数可表述为 $(u, v, 0, \alpha$, $\beta, 0)$ 。

\section{2 几何误差变动约束区间}

滚动导轨直线度公差值为 $T_{F}$, 则直线度误差变 动区间是滚动导轨滚道的曲率中心沿导轨运动方向 形成的曲率圆柱面轴线公差域, 见图 2, 其中 $L$ 是 滑块的长度。

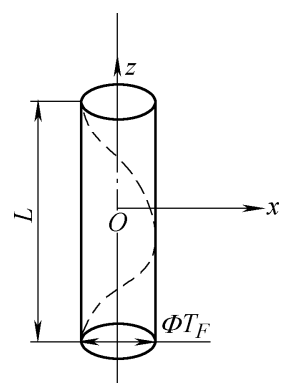

图 2 圆柱面轴线的直线度公差域

直线度公差采用独立原则, 则直线度误差的旋 量参数的变动不等式为

$$
\begin{cases}-\frac{T_{F}}{2} \leqslant u_{F} \leqslant \frac{T_{F}}{2} & -\frac{T_{F}}{2} \leqslant v_{F} \leqslant \frac{T_{F}}{2} \\ -\frac{T_{F}}{L} \leqslant \alpha_{F} \leqslant \frac{T_{F}}{L} & -\frac{T_{F}}{L} \leqslant \beta_{F} \leqslant \frac{T_{F}}{L}\end{cases}
$$

式中, $u_{F}, v_{F}, \alpha_{F}, \beta_{F}$ 为直线度公差的旋量参数。 实际上, 各种旋量参数之间存在约束关系, 即旋量 参数组成的直线度误差沿着导轨运动方向的变动范 围在公差域 $T_{F}$ 内, 其约束不等式为

$$
\left\{\begin{array}{l}
-\frac{T_{F}}{2} \leqslant u_{F}+z \cdot \beta_{F} \leqslant \frac{T_{F}}{2} \\
-\frac{T_{F}}{2} \leqslant v_{F}+z \cdot \alpha_{F} \leqslant \frac{T_{F}}{2}
\end{array}\right.
$$

式中, $z \in[-L / 2, L / 2]$ 。约束不等式(2)的存在缩小了旋
量参数在式(1)中的变化区间。

同时, 滚动导轨还有尺寸误差, 分别是滑块和 导轨滚道半径的尺寸误差等, 其旋量参数为 $\left(u_{C}, v_{C}, 0\right.$, $0,0,0)$ 。尺寸误差与直线度误差变动类似, 在几何 公差 $T_{C}$ 区间内变动, 则滚动导轨尺寸误差旋量参数 $u_{C}, v_{C}$ 的变动不等式为

$$
-\frac{T_{C}}{2} \leqslant u_{C} / \cos \gamma \leqslant \frac{T_{C}}{2} \quad-\frac{T_{C}}{2} \leqslant v_{C} / \sin \gamma \leqslant \frac{T_{C}}{2}
$$

式中, $\gamma$ 为滑块与导轨曲率中心所在方向与水平方 向的角度。

\section{3 基于 MCS 模拟不确定的几何误差}

几何误差旋量参数在确定之后的变动约束区间 内随机分布, 采样统计方法常常用来求解区间约束 下的不确定随机变量。MCS 方法是一种通过对随机 变量的统计试验和随机模拟来求解问题近似解的方 法 $^{[19]}$, 具有适应性强、计算方法简单、求解误差与 问题维数无关等优点。运用 MCS 方法, 对几何误 差旋量参数进行抽样试验, 生成相应的参数随机数 模拟直线度误差, 然后保留满足约束条件的随机数, 去除不满足约束条件的随机数, 并以此作为求解机 床运动误差的根据。

以滚动导轨直线度几何误差为例, 采用 $\mathrm{MCS}$ 方法模拟直线度误差实际变动区间的步骤如下。

(1) 确定几何公差旋量参数的理想概率分布模 型。在滚动导轨的加工和制造过程中, 其几何误差 的大小和方向是不规则的, 由许多相互独立的随机 因素互相影响, 因此其分布情况符合正态分布的分 布规律 ${ }^{[20]}$ 。假定其概率密度函数为

$$
\varphi(x)=\frac{1}{\sigma \sqrt{2 \pi}} \exp \left[\frac{-(x-\mu)^{2}}{2 \delta^{2}}\right] \quad-\infty<x<+\infty \quad \sigma>0
$$

式中, $\mu$ 为算术平均值; $\sigma$ 为均方差。

(2) 确定各旋量参数理想分布的均值与方差。 计算可知概率密度函数中 $x$ 的值出现在 $\mu \pm 3 \sigma$ 区间内 的概率为 $99.73 \%$, 从工程的角度通常认为正态分布 的分布范围是 $\pm 3 \sigma$ 。根据旋量变动不等式 $(1)$, 旋量 参数 $u_{F}, v_{F}, \alpha_{F}, \beta_{F}$ 理想分布的均值与方差分别为 $\left(0, T_{F} / 6\right),\left(0, T_{F} / 6\right),\left(0, T_{F} / 3 L\right),\left(0, T_{F} / 3 L\right)$ 。

(3) 根据抽样要求对几何公差旋量参数进行抽 样, 旋量参数有两种变动顺序 $\left(u_{F}, v_{F} \longrightarrow \beta_{F}, \alpha_{F} ; \alpha_{F}\right.$, $\left.\beta_{F} \rightarrow v_{F}, u_{F}\right)$ 。以变动顺序 $\left(u_{F}, v_{F} \rightarrow \beta_{F}, \alpha_{F}\right)$ 为例, 总的 抽样流程如图 3 所示, 采样样本总数为 $M^{4}$ 。

(4) 为保证模拟精度, 按照两种顺序分别进行 抽样, 可以得到总量为 $2 M^{4}$ 的直线度旋量参数实 际变动区间带宽样本。这些样本用于机床运动误 差分析。 


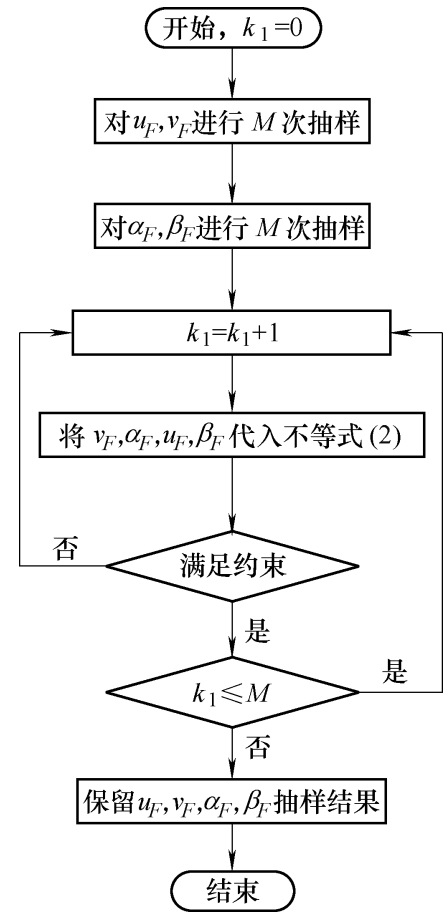

图 3 抽样流程

同理, 采用 MCS 方法模拟滑块和导轨滚道半 径尺寸误差实际变动区间。

\section{2 滚动导轨运动误差建模}

滚动导轨运动误差反映为滑块运动时实际位姿 状态与理想位姿之间连续变化的偏差。任意时刻, 滑块在外载荷、自身重力以及滚动体弹性回复力作 用下处于平衡状态。滚动导轨的几何误差与滑块的 位姿误差会改变滑块、导轨滚动与滚动体的接触状 态, 进而影响滚动体的弹性变形量, 因此本节将滑 块的位姿误差与滚动导轨几何误差等效为滚动体的 弹性变形, 并结合赫兹接触理论建立滚动导轨运动 误差的静力平衡方程, 得到几何误差不确定下滚动 导轨运动误差的分布模型。

\section{1 运动误差描述}

滚动导轨运动误差可以用滑块的位姿误差表 示, 滑块在导轨上某一位置的位姿误差是由此位置 滚动导轨的几何误差和外载荷作用下的弹性变形综 合引起的。滑块相对于理想位置存在 5 个自由度误 差(忽略滑块沿着导轨运动方向的位置误差), 即为 沿着 $X$ 方向的水平位移误差 $\delta_{x}$ 、沿着 $Y$ 方向的坚直 位移误差 $\delta_{x}$ 、绕 $X$ 轴的角度误差 $\varepsilon_{x}$ 、绕 $Y$ 轴的角度 误差 $\varepsilon_{y}$ 和绕 $Z$ 轴的角度误差 $\varepsilon_{z}$ 。考虑误差值一般较 小, 则滑块的位姿误差变换矩阵 $\Delta \boldsymbol{T}_{s}$ 可以描述为

$$
\Delta \boldsymbol{T}_{s}=\left(\begin{array}{cccc}
1 & -\varepsilon_{z} & \varepsilon_{y} & \delta_{x} \\
\varepsilon_{z} & 1 & -\varepsilon_{x} & \delta_{y} \\
\varepsilon_{y} & \varepsilon_{x} & 1 & 0 \\
0 & 0 & 0 & 1
\end{array}\right)
$$

以单列四滚道导轨为例, 假如第 $i$ 列滚道第 $j$ 个滚动体所在对应位置滑块滚道的曲率中心坐标是 $\left[x_{i}^{j}, y_{i}^{j}, z_{i}^{j}, 1\right]^{\mathrm{T}}$, 则其位姿误差在水平和坚直方向的 偏差量分别为 $\delta_{x i}^{j}$ 和 $\delta_{y i}^{j}$, 可以表达为

$$
\left\{\begin{array}{l}
\delta_{x i}^{j}=\delta_{x i}+\varepsilon_{y i} \bullet z_{i}^{j}-\varepsilon_{z i} \cdot y_{i}^{j} \\
\delta_{y i}^{j}=\delta_{y i}-\varepsilon_{x i} \cdot z_{i}^{j}+\varepsilon_{z i} \cdot x_{i}^{j}
\end{array}\right.
$$

\section{2 误差等效模型}

滚动导轨几何误差和滑块位姿误差反映为滑块 和导轨滚道曲率中心的偏移量, 因此将几何误差和 滑块位姿误差等效为滚动体的弹性变形, 然后根据 赫兹公式建立滑块的静力平衡方程。理想状态下, 滚动体弹性变形之前的滑块与滚动导轨的接触模型 如图 4 所示。

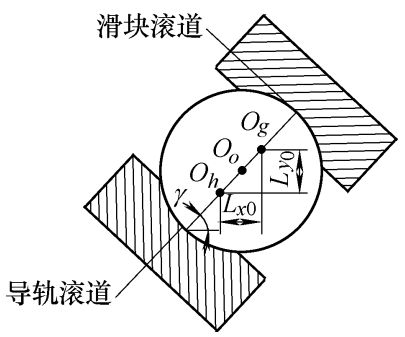

图 4 变形前滚动导轨接触模型

图 4 中, $O_{g}$ 和 $O_{h}$ 分别是导轨和滑块滚道曲率 中心。则导轨滚道的曲率中心与滑块滚道的曲率中 心的距离为

$$
\left|O_{g}-O_{h}\right|=2 f D_{a}-D_{a}=(2 f-1) D_{a}
$$

此距离在 $X$ 和 $Y$ 方向上的分量分别为 $L_{x 0}=$ $(2 f-1) D_{a} \cos \gamma, L_{y 0}=(2 f-1) D_{a} \sin \gamma 。 D_{a}$ 是滚动体公称直 径, $f$ 是滑块和导轨滚道半径与滚动体直径的调整系 数, 即设计时滚道的直径需要略大于滚动体的直径。

滚动体的变形量与滑块和滚道曲率中心的位置 直接相关。理想状态下滚动体的弹性变形是沿滑块和 滚道曲率中心方向的尺寸变化, 实际中导轨滚道曲率 中心水平和坚直方向的偏移量分别为 $\Delta_{i j x}$ 和 $\Delta_{i j y}$; 滑块 滚道曲率中心水平和坚直方向的偏移量分别为 $\delta_{i j x}$ 和 $\delta_{i j y}$, 变形之后滚动导轨的接触模型如图 5 所示。

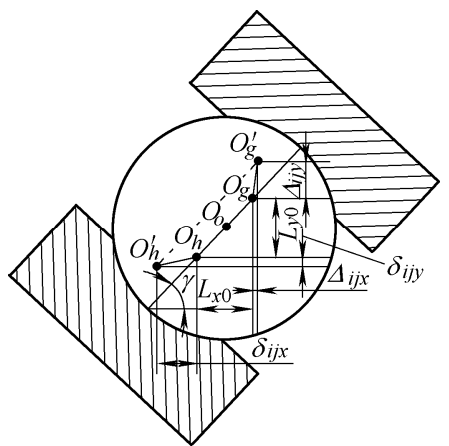

图 5 变形后滚动导轨误差等效模型 
因此, 变形后滚动体的变化量为

$$
\begin{gathered}
\Delta_{i j}=\left|O_{g}^{\prime}-O_{h}^{\prime}\right|-\left|O_{g}-O_{h}\right|=\left(L_{i j x}^{2}+L_{i j y}^{2}\right)^{\frac{1}{2}}-(2 f-1) D_{a} \\
L_{i j x}=L_{x 0}-\delta_{i j x}+\Delta_{i j x} \\
L_{i j y}=L_{y 0}-\delta_{i j y}+S_{i j y}
\end{gathered}
$$

一般情况下, 滚动导轨通过加大滚动体直径直 接预紧。因此, 若滚动体直径增量是 $\lambda$, 其实际变 形量为

$$
\Delta_{i j}=\left(L_{i j x}^{2}+L_{i j y}^{2}\right)^{\frac{1}{2}}-(2 f-1) D_{a}+\lambda
$$

\section{3 导轨运动误差求解}

基于赫兹接触理论的弹性回复力与其弹性变形 之间的关系, 建立滑块在外载荷、重力以及所有滚 动体回复力下的力和力矩平衡方程。根据赫兹接触 公式, 滚动导轨中单一滚动体弹性变形 $\Delta$ 与弹性回 复力大小 $P$ 之间的关系为

$$
P=(2 \eta)^{-3 / 2} \Delta^{3 / 2}
$$

式中, $\eta$ 为与滚动体直径和滚道曲率有关的接触系 数 ${ }^{[21]}$ 。由式(9)、(10)可以得到滚动直线导轨的接触 力 $P_{i j}$, 在 $X$ 和 $Y$ 方向上的接触分力

$$
P_{i j x}=P_{i j} \cdot \cos \beta_{i j}, P_{i j y}=P_{i j} \cdot \sin \beta_{i j}
$$

式中, $\beta_{i j}$ 是变形之后的接触角, $\beta_{i j}=\arctan \left|L_{i j,} / L_{i j k}\right|$ 。 对于滑块而言, 各个滚动体到滑块坐标原点的矢径 可以记为 $r_{i j}\left(r_{i j}=\left(X_{i j}, Y_{i j}, Z_{i j}\right)\right)$, 因此滑块中所有滚 动体的接触力以及合力矩可以表达为

$$
\begin{gathered}
F_{x}=\sum_{i=1}^{4} \sum_{j=1}^{n} P_{i j x} \\
F_{y}=\sum_{i=1}^{4} \sum_{j=1}^{n} P_{i j y} \\
M_{x}=\sum_{i=1}^{4} \sum_{j=1}^{n} P_{i j y} Z_{i j} \\
M_{y}=\sum_{i=1}^{4} \sum_{j=1}^{n} P_{i j x} Z_{i j} \\
M_{z}=\sum_{i=1}^{4} \sum_{j=1}^{n} P_{i j x} Y_{i j}+\sum_{i=1}^{4} \sum_{j=1}^{n} P_{i j y} X_{i j}
\end{gathered}
$$

滑块所受外力为自身重力、外载荷及所有滚动 体的弹性支反力, 并处于平衡状态, 即滑块弹性支 反力及其合力矩与所受外力和外力矩的平衡方程为

$$
\begin{gathered}
\sum_{i=1}^{4} \sum_{j=1}^{n} P_{i j x}+F_{o u t x}=0 \\
\sum_{i=1}^{4} \sum_{j=1}^{n} P_{i j y}+F_{o u t y}+G_{p a r t}=0
\end{gathered}
$$

$$
\begin{gathered}
\sum_{i=1}^{4} \sum_{j=1}^{n} P_{i j y} Z_{i j}+M_{\text {outx }}=0 \\
\sum_{i=1}^{4} \sum_{j=1}^{n} P_{i j x} Z_{i j}+M_{\text {outy }}=0 \\
\sum_{i=1}^{4} \sum_{j=1}^{n} P_{i j x} Y_{i j}+\sum_{i=1}^{4} \sum_{j=1}^{n} P_{i j y} X_{i j}+M_{\text {outz }}=0
\end{gathered}
$$

基于得到的滚动导轨几何误差旋量参数样本, 联合式(6) (21), 可以求解在滚动导轨任意位置的 运动误差参数。

\section{3 实例求解与分析}

根据上述滚动导轨运动误差模型, 探究导轨直 线度公差约束、预紧力以及外载荷对导轨运动误差 的影响规律。

\section{1 滚动导轨结构参数}

以某单列四滚道的滚动导轨为例, 滑块行程为 $1200 \mathrm{~mm}$, 滚动导轨结构参数如表 1 所示。

表 1 滚动导轨的结构参数

\begin{tabular}{lc}
\hline \multicolumn{1}{c}{ 参数 } & 数值 \\
\hline 导轨数量 & 1 \\
单列滚动体数量 & 8 \\
滚动体直径 $/ \mathrm{mm}$ & 10.0 \\
初始滚动体接触角 $/\left(^{\circ}\right)$ & 45 \\
滑块的宽度 $/ \mathrm{mm}$ & 80.0 \\
滑块的高度 $/ \mathrm{mm}$ & 45.6 \\
滑块的长度 $/ \mathrm{mm}$ & 100.0 \\
\hline
\end{tabular}

\section{2 预紧力对滑块位姿误差的影响}

通常情况下滚动导轨需要施加预紧力以消除间 隙、提高导轨的刚性, 如加大滚动体直径。根据产 品样本手册, 选取轻预紧力、中预紧力和重预紧力 三种预紧情况, 分析预紧力对滚动导轨运动误差的 影响。在滑块和滚道曲率半径尺寸公差为 $0.01 \mathrm{~mm}$, 导轨的直线度公差为 $0.05 \mathrm{~mm}$ 内采样 10000 个点, 分析不同预紧下滑块的五自由度位姿误差分布规 律, 如图 6 所示。图中可以看出预紧力对滑块位姿 误差有显著影响, 尤其是沿着 $X$ 方向的侧向误差和 绕 $Z$ 轴的滚摆误差。图 6a、6b 中可以看出, 随着预 紧力的增加, 滑块沿 $X$ 方向的侧向误差从最大的 $0.06 \mathrm{~mm}$ 减小到 $0.03 \mathrm{~mm}$; 绕 $Z$ 轴的滚摆误差从 2 $\mathrm{mrad}$ 减少到 $0.8 \mathrm{mrad}$; 但滑块沿着 $Y$ 方向的坚直误 差分布并没有明显变化。因此, 从轻预紧力到重预 
紧力滑块侧向误差减小了 $0.03 \mathrm{~mm}$ 、滚摆误差减小 了 $0.8 \mathrm{mrad}$ 。

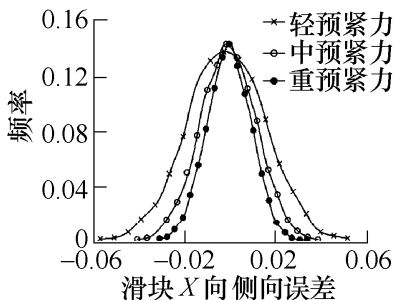

(a) 滑块 $X$ 向侧向误差

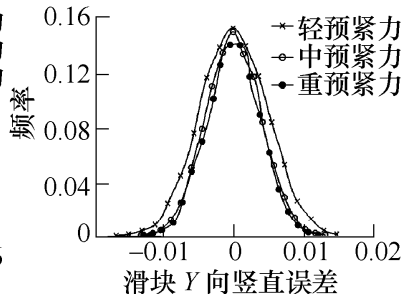

(b) 滑块 $Y$ 向坚直误差

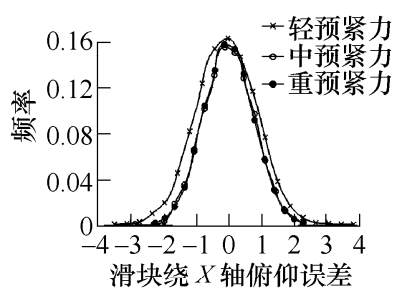

(c) 滑块绕 $X$ 轴俯仰误差

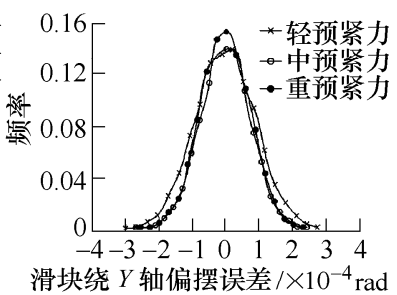

(d) 滑块绕 $Y$ 轴偏摆误差

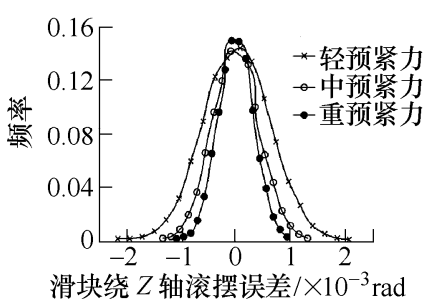

(e) 滑块绕 $Z$ 轴滚摆误差

图 6 不同预紧力下滑块位姿误差

\section{3 直线度公差对滑块位姿误差的影响}

滚动导轨分为普通级、高级和精密级三种不同 精度等级。经过计算, 则不同高级等级下滑块的五 自由度位姿误差分布规律如图 7 所示。从分析结果 可以看出随着精密等级的提高, 滑块的五自由度位 姿误差都有明显减小趋势，而且分布范围更小。当 滑块直线度公差从 $0.05 \mathrm{~mm}$ 减小到 $0.01 \mathrm{~mm}$ 时, 滑 块沿 $X$ 方向的侧向误差从 $0.06 \mathrm{~mm}$ 减小到 $0.01 \mathrm{~mm}$; 沿 $Y$ 方向的坚直误差从 $15 \mu \mathrm{m}$ 减小到 $1 \mu \mathrm{m}$, 变化非 常显著; 绕 $X$ 轴的俯仰误差从 $0.4 \mathrm{mrad}$ 减小到 0.05 $\mathrm{mrad}$; 绕 $Y$ 轴的偏摆误差从 $0.4 \mathrm{mrad}$ 减小到 0.05 $\mathrm{mrad}$; 绕 $Z$ 轴的滚摆误差从 $2 \mathrm{mrad}$ 减小到 $0.3 \mathrm{mrad}$, 变化非常明显。

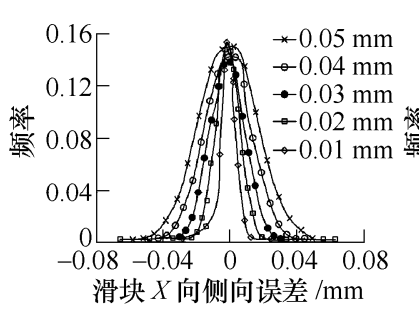

(a) 滑块 $X$ 向侧向误差

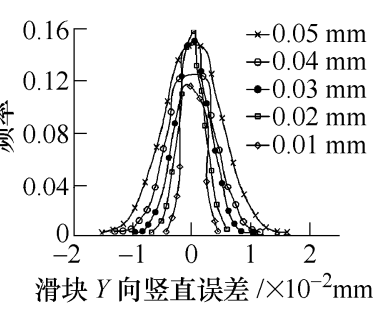

(b) 滑块 $Y$ 向坚直误差
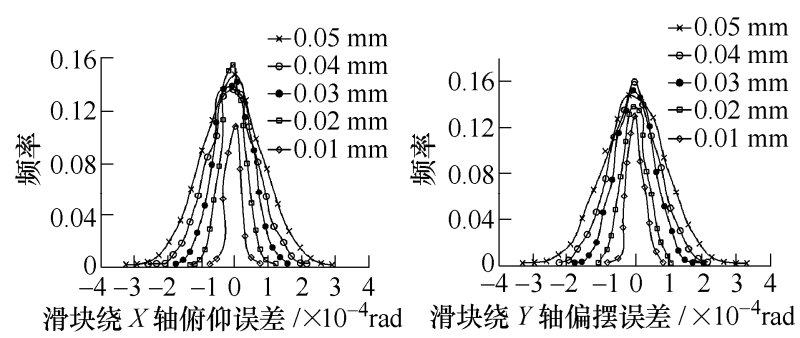

(c) 滑块绕 $X$ 轴俯仰误差

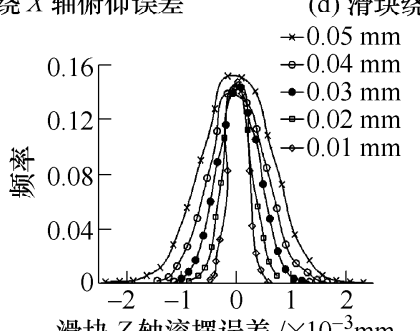

滑块 $Z$ 轴滚摆误差 $/ \times 10^{-3} \mathrm{~mm}$

(e) 滑块绕 $Z$ 轴滚摆误差

图 7 不同直线度公差下滑块位姿误差

\section{4 外载荷对滑块位姿误差的影响}

在坚直方向不同外载荷作用下的滑块姿误差分 布规律如图 8 所示。从图 8 中可以看出随着外载荷 的增大, 滑块沿 $X$ 方向的侧向误差和绕 $X$ 轴的俯仰 误差整体有明显减小的趋势，但是误差分布的宽度 和范围并没有明显变化。 $X$ 方向的侧向误差分布范 围从-0.06 0.05 mm 变为- $-0.07 \sim 0.04 \mathrm{~mm}$ 、绕 $X$ 轴 的俯仰误差从 $-0.4 \sim 0.4 \mathrm{mrad}$ 变为 $-0.5 \sim 0.2 \mathrm{mrad}$; 沿 $Y$ 方向的坚直误差和绕 $Z$ 轴的滚摆误差有整体增

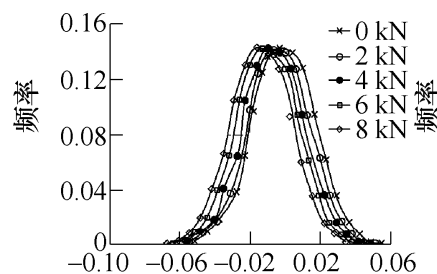

滑块 $X$ 向侧向误差 $/ \mathrm{mm}$

(a) 滑块 $X$ 向侧向误差

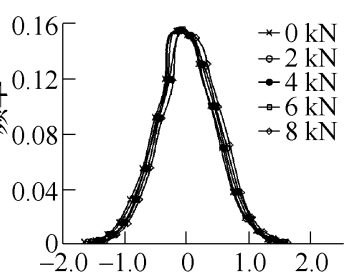

滑块 $Y$ 向坚直误差 $/ \times 10^{-3} \mathrm{~mm}$
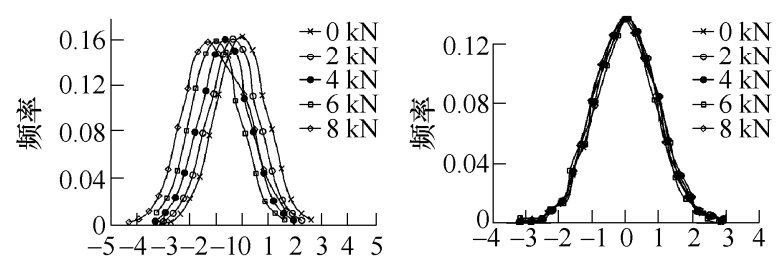

滑块绕 $X$ 轴俯仰误差 $/ \times 10^{-4} \mathrm{rad}$ 滑块绕 $Y$ 轴角度误差 $/ \times 10^{-4} \mathrm{rad}$ (c) 滑块绕 $X$ 轴俯仰误差

(d) 滑块绕 $Y$ 轴偏摆误差

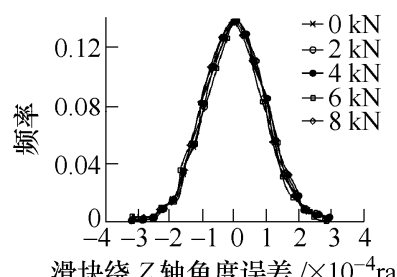

滑块绕 $Z$ 轴角度误差 $/ \times 10^{-4} \mathrm{rad}$

(e) 滑块绕 $Z$ 轴滚摆误差

图 8 不同外载荷下滑块位姿误差 
大的趋势, 但是变化并不是很明显; 绕 $Y$ 轴的偏 摆误差变化不是很大。可以看出, 滑块位姿误差 的分布范围对外载荷的敏感性不是很高, 整体变 化不明显。

\section{4 结论}

（1）基于不确定性分析方法, 采用区间参数和 小位移旋量实现了设计阶段不确定的滚动导轨误差 描述, 并采用 MCS 方法完成了不确定误差模拟仿 真。

(2) 将滚动导轨不确定的几何误差和滑块位姿 误差等效为滚动体的弹性变形, 基于赫兹接触理论 建立滚动导轨的静力平衡方程, 获得了运动误差分 析模型。

(3) 运动误差分析结果表明: 提高预紧力可以 明显改善滑块侧向误差和滚摆误差; 随着导轨直线 度公差的减小, 滑块位姿误差明显减小, 分布范围 更小、更密集; 外载荷的增加可以减小滑块侧向误 差和俯仰误差、增大滑块坚直误差和滚摆误差, 但 对它们的分布范围影响不是很大。

\section{参 考 文 献}

[1] 孙永平. 机床静态精度的机构模型与结构工艺性研究 [D]. 大连: 大连理工大学, 2015.

SUN Yongping. Research on the mechanism model and structure processing of machine tool accuracy[D]. Dalian: Technology of Dalian University, 2015.

[2] 程强, 刘广博, 刘志峰, 等. 基于敏感度分析的机床关 键性几何误差源识别方法[J]. 机械工程学报, 2012, 48(7): 171-179.

CHENG Qiang, LIU Guangbo, LIU Zhifeng, et al. An identification approach for key geometric error sources of machine tool based on sensitivity analysis[J]. Journal of Mechanical Engineering, 2012, 48(7): 171-179.

[3] 胡鹏浩, 张建坤, 马晓庆, 等. 3-PUU 并联测量机滑块 运动误差分析 $[\mathrm{J}]$. 机械工程学报，2015，51(18): 1-6. HU Penghao, ZHANG Jiankun, MA Xiaoqing, et al. Analysis of slider motion error on 3-PUU parallel coordinate measuring maching[J]. Journal of Mechanical Engineering, 2015, 51(18): 1-6.

[4] 要小鹏, 殷国富, 李光明. 数控机床进给轴综合误差解 耦建模与补偿研究 [J]. 机械工程学报, 2016, 52(1): 184-192.

YAO Xiaopeng, YIN Guofu, LI Guangming. Positioning error of feed axis decouple-separating modeling and compensating research for $\mathrm{CNC}$ machine tools[J]. Journal of Mechanical Engineering, 2016， 52(1): 184-192.

[5] OHTA H, TANAKA K. Vertical stiffness of preloaded linear guideway type ball bearing incorporating the flexibility of the carriage and rail[J]. Journal of Tribology-Transactions of the ASME, 2010, 132(1): 1-9.

[6] JEONG J, KANG E, JEONG J. Equivalent stiffness modeling of linear motion guideways for stage systems $[\mathrm{J}]$. International Journal of Precision Engineering and Manufacturing, 2014, 15(9): 1987-1993.

[7] KHIM G, OH J S, PARK C H. Analysis of 5-DOF motion errors influenced by the guide rails of an aerostatic linear motion stage $[\mathrm{J}]$. International Journal of Precision Engineering and Manufacturing, 2014, 15(2): 283-290.

[8] KHIM G, PARK C H, OH J S. A method of calculating motion error in a linear motion bearing stage[J]. The Scientific World Journal, 2015: 696417.

[9] RACKWITZ R, FIESSLER B. Structural reliability under combined random load sequences[J]. Computers \& Structures, 1978, 9(5): 489-494.

[10] YANG Z Z. Reliability-based robust optimization design of automobile components with non-normal distribution parameters $[\mathrm{J}]$. Chinese Journal of Mechanical Engineering, 2013, 26(4): 823-830.

[11] 刘成武, 李连升, 钱林方. 随机与区间不确定下基于近 似灵敏度的序列多学科可靠性设计优化 [J]. 机械工程 学报, 2015, 51(21): 174-184.

LIU Chengwu, LI Liansheng, QIAN Linfang. Sequential multidisciplinary reliability design and optimization based on approximate sensitivity method under random and interval uncertainties[J]. Journal of Mechanical Engineering, 2015, 51(21): 174-184.

[12] 吕辉，于德介，陈宁，等. 基于可靠性的混合不确定参 数汽车盘式制动器振动稳定性分析 $[\mathrm{J}]$. 机械工程学报, 2014, 50(6): 112-119.

LÜ Hui, YU Dejie, CHEN Ning, et al. Reliability analysis for vibration stability of automotive disc brake system with hybrid uncertain parameters[J]. Journal of Mechanical Engineering, 2014， 50(6): 112-119.

[13] 黎凯, 杨旭静, 郑娟. 基于参数和代理模型不确定性的 冲压稳健性设计优化 [J]. 中国机械工程, 2015，26(23): 3234-3239.

LI Kai, YANG Xujing, ZHENG Juan. Robust design optimization for stamping based on parametric and metamodel uncertainty[J]. China Mechanical Engineering, 2015, 26(23): 3234-3239.

[14] 熊芬芬. 稳健优化设计中代理模型不确定性的研究 [J]. 机械工程学报, 2014, 50(19): 136-143.

XIONG Fenfen. Robust design optimization considering metamodel uncertainty[J]. Journal of Mechanical 
Engineering, 2014, 50(19): 136-143.

[15] 余治民, 刘子建, 董思科, 等. 基于蒙特卡洛模拟与响 应面方法的公差建模[J]. 中国机械工程, 2015, 26(4): 427-434

YU Zhimin, LIU Zijian, DONG Sike, et al. Tolerance modeling based on Monte-Carlo simulation and response surface method[J]. China Mechanical Engineering, 2015, 26(4): 427-434.

[16] 崔金䨝, 李威. 基于蒙特卡洛模拟的 6-UPS 并联机构的 误差分析[J]. 机械设计与制造，2016(6)：89-92.

CUI Xinlei, LI Wei. Error analysis of 6-UPS parallel mechanisms based on Monte Carlo simulation[J]. Machinery Design \& Manufacture, 2016(6): 89-92.

[17] 吴兆强. 基于小位移旋量的公差模拟建模及公差分析 [J]. 机械设计与制造，2010(1)：205-207.

WU Zhaoqiang. Stimulated tolerances modeling based on small displacement torsors and tolerances analysis[J]. Machinery Design \& Manufacture, 2010(1): 205-207.

[18] BOURDET P, MATHIEU L, LARTIGUE C, et al. The concept of the small displacement torsor in metrology[J]. Adv. Math. Tools Metrol., 1996, 40: 110-122.

[19] ZHONG X, YANG R Q, ZHOU B. Accuracy analysis of assembly success rate with Monte Carlo simulations[J]. Journal of Donghua University, 2003，20(4): 128-131.

[20] 贾振元, 王福吉. 机械制造技术基础[M]. 北京: 科学 出版社, 2011.

JIA Zhenyuan, WANG Fuji. Foundation of machine manufacturing technology[M]. Beijing: Science Press, 2011.

[21] MAJDA P. Modeling of geometric errors of linear guideway and their influence on joint kinematic error in machine tools[J]. Precision Engineering, 2012, 36: 369-378.

作者简介: 马雅丽(通信作者), 女, 1963 年出生, 教授, 硕士研究生导 师。主要研究方向为机械系统设计及创新设计理论。

E-mail: myl@dlut.edu.cn

李阳阳, 男, 1991 年出生, 硕士研究生。主要研究方向为机械系统设计 及创新设计理论。

E-mail: liyangying2010@163.com 